\title{
Mifepristone Derivative FZU-00,003 Suppresses Triple-negative Breast Cancer Cell Growth partially via miR-153-KLF5 axis
}

\author{
Rong Liu ${ }^{1,2^{*}}$, Haijun Chen ${ }^{3 *}$, Ping Zhao ${ }^{4}$, Chuan-Huizi Chen ${ }^{1}$, Huichun Liang1, Chuanyu Yang1, Zhongmei \\ Zhou $^{1}$, Xu Zhi ${ }^{5}$, Suling Liu ${ }^{6,7}$, Ceshi Chen ${ }^{1,8}{ }^{\circledR}$ \\ 1. Key Laboratory of Animal Models and Human Disease Mechanisms of the Chinese Academy of Sciences and Yunnan Province, Chinese Academy of \\ Sciences, Kunming Institute of Zoology, Kunming, Yunnan 650223, China; \\ 2. Center for Excellence in Animal Evolution and Genetics, Chinese Academy of Sciences, Kunming, PR China \\ 3. College of Chemistry, Fuzhou University, Fuzhou, Fujian 350108, China; \\ 4. Department of Breast Surgery, Yunnan Cancer Hospital, The Third Affiliated Hospital of Kunming Medical University, Kunming, Yunnan, 650118, China; \\ 5. Center for Reproductive Medicine, Department of Obstetrics and Gynecology, Peking University Third Hospital, Beijing 100191, China; \\ 6. Key Laboratory of Breast Cancer in Shanghai, Cancer Institute, Department of Breast Surgery, Fudan University Shanghai Cancer Center, Shanghai 200032, \\ China; \\ 7. Institutes of Biomedical Sciences, Fudan University, Shanghai 200032, China; \\ 8. KIZ-CUHK Joint Laboratory of Bioresources and Molecular Research in Common Diseases, Kunming Institute of Zoology, Chinese Academy of Sciences, \\ Kunming, 650223, China. \\ *These authors contribute equally to this study. \\ $\bowtie$ Corresponding author: Ceshi Chen, Tel: 86-871-5181944, E-mail: chenc@mail.kiz.ac.cn.
}

(c) The author(s). This is an open access article distributed under the terms of the Creative Commons Attribution License (https://creativecommons.org/licenses/by/4.0/). See http://ivyspring.com/terms for full terms and conditions.

Received: 2019.08.20; Accepted: 2019.11.12; Published: 2020.01.01

\begin{abstract}
Triple-negative breast cancer (TNBC) is one of the most malignant breast cancers lacking targeted therapeutics currently. We recently reported that mifepristone (MIF), a drug regularly used for abortion, suppresses TNBC cell growth by inhibiting KLF5 expression via inducing miR-153. However, its anticancer efficacy is only modest at high dose. In order to enhance the anticancer activities, a focused compound library containing 17 compounds by altering the sensitive metabolic region of mifepristone has been designed and synthesized. We first tested the cell growth inhibitory effects of these compounds in TNBC cell lines. Among them, FZU-00,003 displayed the most potent efficiency. FZU-00,003 suppresses TNBC cell growth, cell cycle progression and induces apoptosis more effectively than MIF does. Consistently, FZU-00,003 induces miR-153 expression and suppressed KLF5 expression at much lower dosages than MIF does. Furthermore, FZU-00,003 inhibits tumor growth more potently than MIF does. Taken together, the MIF derivative, FZU-00,003 may serve as a better therapeutic compound for TNBC than MIF.
\end{abstract}

Key words: FZU-00,003, KLF5, breast cancer, MIF derivatives

\section{Introduction}

Classically, breast cancer can be divided into three subtypes, including estrogen receptor (ER $\alpha)$ positive w/o progesterone receptor (PR) positive luminal subtype, human epidermal growth factor receptor 2 (HER2) positive HER2 subtype, and ER-/PR-/HER2- triple-negative subtype (TNBC). Among these subtypes, TNBC constitutes $10 \%-20 \%$ of all breast cancers, and is usually of higher grade and biologically more aggressive [1]. TNBC has been challenging due to the heterogeneity of the disease, the absence of well-defined molecular targets and effective targeted therapies [2]. Therefore, the adjuvant chemotherapy is the only choice. Even so, the prognosis of TNBC is not optimistic, more than $70 \%$ of women with metastatic TNBC die within 5 years [3]. Thus, it is urgently needed to discover new targets and develop mechanism-based, effective targeted therapies for this aggressive type of breast 
cancer [4]. Although efforts have been made to achieve this goal by numerous investigations, such as PARP1 inhibitors [5], EGFR inhibitors [6], mTOR inhibitors [7], and bromodomain inhibitors [8,9] have been reported to display anticancer efficacy in TNBC models. Despite some initial success, most of the agents have shown only limited efficacy in both preclinical and clinical settings. Therefore, only a few of them are advanced into clinical trials for the treatment of TNBC patients [10].

Human Krüppel-like factor 5 (KLF5) has been implicated in promoting breast cell proliferation, survival, stemness and tumorigenesis [11-14]. Data from clinical samples indicated that KLF5 is highly expressed in basal-type breast tumors [15], and high levels of KLF5 are positively correlated with shorter survival for breast cancer patients [16, 17]. We previously identified that KLF5 is specifically highly expressed in basal TNBC cell lines [18], and depletion of KLF5 significantly suppresses basal TNBC cell proliferation, survival and tumor growth $[19,20]$. Our recent work implicated that pharmacological inhibition of KLF5 could suppress TNBC cell growth $[13,14]$. These data implicated that KLF5 could serve as a promising target for TNBC.

Recently, we found mifepristone (MIF), a synthetic progesterone receptor (PR) antagonist, which has been widely used as an abortifacient and emergency contraceptive for decades [21], suppresses TNBC cell proliferation and tumor growth partially through inhibiting KLF5 expression via inducing miR-153 [13]. Although MIF shows anti-TNBC activity, the potency is relatively moderate (with a half maximal inhibitory concentration $\left(\mathrm{IC}_{50}\right)$ of $10-22$ $\mu \mathrm{M}$ for TNBC cells), which has limited its further clinical application for TNBC treatment [13].

To develop more potent anti-TNBC reagents, we designed and synthesized 17 MIF derivatives, and compared the anticancer efficacy of MIF and its derivatives. We found several analogs suppressed TNBC cell viability more potently than MIF did, and FZU-00,003 had the lowest $\mathrm{IC}_{50}$ when compared to other derivatives. Furthermore, we demonstrated that FZU-00,003 suppressed TNBC cell growth, cell cycle progression and induced apoptosis more effectively than MIF did. FZU-00,003 induced miR-153 expression and suppressed KLF5 expression at much lower dosages than MIF does. Finally, we evaluated the anticancer efficacy of FZU-00,003 in a patient-derived xenograft model and found FZU-00,003 inhibited tumor growth more potently than MIF did. Taken together, our data suggest that the MIF derivative, FZU-00,003 may serve as a better lead compound for TNBC than MIF.

\section{Materials and methods}

\section{Cell Culture and Transfection}

All cell lines were purchased from the American Type Culture Collection (ATCC) and were validated by the STR analysis (Kunming Cell Bank, Kunming Institute of Zoology, Chinese Academy of Sciences). HCC1937 and HCC1806 were cultured in RPMI-1640 medium (HyClone, Logan, UT) supplemented with $5 \%$ fetal bovine serum (FBS). SUM149PT was cultured with Ham's F-12 medium (HyClone, Logan, UT) supplemented with 5\% FBS, $0.5 \mu \mathrm{g} / \mathrm{ml}$ hydrocortisone and $10 \mu \mathrm{g} / \mathrm{ml}$ insulin. T47D and BT474 cell lines were maintained with RPMI-1640 medium supplemented with 5\% FBS and $10 \mu \mathrm{g} / \mathrm{ml}$ insulin. MCF7 and SKBR3 cells were cultured in DMEM medium with 5\% FBS. All cells were maintained at $37{ }^{\circ} \mathrm{C}$ with $5 \% \mathrm{CO}_{2}$ in a humidified atmosphere.

MicroRNA inhibitor (Ribobio, Guangzhou, China) was transfected into different cell lines using Lipofectamine 2000 following the manufacturer's manual (Invitrogen, Carlsbad, CA, USA).

\section{Cell Viability Assays}

Cell viability was quantified with the sulforhodamine B (SRB) assay as described previously $[13,22]$. Briefly, $1-4 \times 10^{4}$ cells per well were plated in 48-well plates and treated with mifepristone, mifepristone derivatives or DMSO at designed concentrations for 48 hours. The cells were then fixed with $10 \%$ trichloroacetic acid at $4{ }^{\circ} \mathrm{C}$ overnight followed by washing with deionized water 5 times. The cells were stained with $0.4 \%(\mathrm{~W} / \mathrm{V}) \mathrm{SRB}$ in $1 \%$ acetic acid for $15 \mathrm{~min}$ at room temperature. The plates were washed 5 times with $1 \%$ acetic acid and dried. Finally, $10 \mathrm{mM}$ Tris base was added to dissolve the dye and the optical densities at $530 \mathrm{~nm}$ were determined at a spectrophotometric plate reader (Epoch, Bio-Tek, USA).

\section{Cell Proliferation Assay}

Cell proliferation of SUM149PT and HCC1937 cells was measured using Click-iT EdU Alexa Fluor 488 Imaging Kit (Invitrogen) following the manufacturer's instructions. Briefly, drug treated cells were incubated with EdU solution for 4 hours before being fixed using 3.7\% formaldehyde. After washing with 3\% BSA (bovine serum albumin), cells were permeabilized by $0.5 \%$ Triton X-100 (Sigma) and incorporated EdU were detected by Alexa Fluor azide. Nuclei were stained with Hoechst 33342. The total numbers of cells and EdU-incorporated cells in each sample were counted using a fluorescent microscope. 


\section{Cell Cycle Analysis}

Drug or DMSO treated cells were trypsinized, harvested, washed once with $1 \times$ PBS and fixed with $70 \%$ ethanol at $4{ }^{\circ} \mathrm{C}$ overnight. Fixed cells were washed with $1 \times$ PBS and stained with propidium iodide (PI) buffer $(0.05 \mathrm{mg} / \mathrm{ml}$ PI, $0.3 \%$ NP-40, $0.5 \mathrm{mg} / \mathrm{ml}$ RNase A) for $30 \mathrm{~min}$ in dark at room temperature and subjected to cell cycle analysis via Accuri $^{\mathrm{TM}}$ C6 flow cytometry (BD bioscience, San Diego, CA) within 4 hours.

\section{Apoptosis Analysis}

Trypsinized cells were collected and washed by $1 \times$ PBS once. Cells were counted and $1 \times 10^{5}$ cells stained with anti-Annexin $\mathrm{V}$ antibody and propidium iodide following the manufacturer's protocol (eBioscience, San Diego, CA). Briefly, the cells were washed with $1 \times$ binding buffer followed by staining with FITC-labeled annexin V in dark for 30 min at RT. Following annexin $\mathrm{V}$ staining, the cells were washed with $1 \times$ binding buffer twice, stained with propidium iodide, and analyzed on the Accuri C6 flow cytometer (BD bioscience).

\section{Western Blot Analysis and Antibodies}

Cell lysates were prepared as described previously [23]. Briefly, cells were collected in cell lysis buffer for protein extraction, and $40 \mu \mathrm{g}$ protein samples were subjected to SDS-PAGE and blotted onto polyvinylidene fluoride (PVDF) membranes. Membranes were blocked with 5\% non-fat milk for 1 hour at RT and then incubated with the specific primary antibodies at $4^{\circ} \mathrm{C}$ overnight. After washing with PBS containing 0.1\% Tween-20 (PBST), the membranes were incubated with horseradish peroxidase (HRP) conjugated secondary antibodies (Jackson ImmunoResearch Laboratory, West Grove, PA) for 1 hour at RT. The Membranes were washed with PBST and incubated with Western Lighting Chemiluminescence Reagent Plus (PerkinElmer Life Sciences, Shelton, CT) and then the targeted proteins were visualized on an ImageQuant LAS4000 Biomolecular imager (GE, PA).

The anti-PARP and p21 antibodies were purchased from Cell Signaling Technology (Danvers, MA). The anti-p27 antibody was from Becton Dickinson (San Diego, CA).The anti-KLF5 rabbit polyclonal antibody has been described previously [23].The anti- $\beta$-actin antibody was from Sigma (St. Louis, MO).

\section{microRNA Reverse-transcription and qPCR}

HCC1937 and SUM149PT cells were treated with FZU-00,003, MIF or DMSO control at indicated dosages for 24 hours. Total mRNAs were isolated using TRIzol ${ }^{\circledR}$ reagent (Invitrogen). Reverse transcription was performed using the TaqMan ${ }^{\circledR}$ MicroRNA Reverse Transcription Kit (Thermo Fisher Scientific, Fremont, CA) and miRNA levels were quantified using RT Real-Time ${ }^{\mathrm{TM}}$ SYBR Green/Rox PCR master mix (SAbiosciences, CA) on the ABI-7900 system. Primers for U6 and miR-153 are listed in supplementary table 1.

\section{Stable Over-expression of KLF5}

The KLF5 cDNA was amplified and cloned into pCDH-puro lentiviral vector using forward primer, 5'-AGAGAATTCGGATCCATGGCTACAAGGGTGC TG-3' and reverse primer 5'-CTTCCATGGCTCGAGT CAGTTCTGGTGCCTCTTC-3'. To prepare the lentivirus, all lentiviral plasmids and the packing plasmids were co-transfected into HEK293T cells using Lipofectamine 2000. Lentiviruses were collected at 72 hours after transfection and used to transduce HCC1937 cells in 6-well plates. 24 hours after transduction, puromycin $(1 \mu \mathrm{g} / \mathrm{ml})$ was added to select drug-resistant cell populations.

\section{Tumorigenesis in BALB/c Nude Mice}

This study was approved by the institutional ethics committees of Kunming Institute of Zoology, Chinese Academy of Sciences. Eighteen 6-week-old female BALB/c nude mice were purchased from SJA Laboratory Animal Co., Ltd. (Hunan, China). The ER $\alpha-P R-H E R 2-$ invasive ductal carcinoma UM1 patient-derived xenograft (PDX) tissues, which were maintained in fat-pads of BALB/c nude mice without culture in vitro, were collected and dissociated as described previously [13]. UM1 cell suspension $\left(1 \times 10^{6}\right.$ cells/point) was implanted into mammary fat pads of the BALB/c nude mice. Tumor size was measured using Vernier calipers once tumors became palpable. Tumor volumes were calculated using the following equation: tumor volume $\left(\mathrm{cm}^{3}\right)=\Pi \times$ (length $\times$ width $\left.{ }^{2}\right) / 6$. When the tumor size reached $50 \mathrm{~mm}^{3}$, mice were randomly and equally distributed into three groups, which were treated with the vehicle control, 1 mg of FZU-00,003 or $1 \mathrm{mg}$ of mifepristone via daily intraperitoneal injections. Tumor size was monitored twice/week for 4 weeks. All mice were sacrificed at the end of the experiment and tumors were harvested for analysis.

\section{Statistical Analysis}

The cell cycle assay, cell apoptosis analysis and the cell viability assay were conducted in triplicate. When appropriate, the data were pooled to generate means \pm standard deviation and were analyzed by t-test. P-values less than 0.05 were considered to be significant. 


\section{Results}

\section{Several MIF derivatives decreases TNBC cell viability more potently than MIF}

In order to develop more potent MIF based anti-cancer reagents, we synthesized 17 analogs based on the chemical structure of mifepristone by altering the sensitive metabolic region (Supplementary Table 2). Our preliminary screening results (Fig. 1A) revealed that $\mathrm{N}$-monodemethyl mifepristone (FZU00,001 ) without methyl moiety is not favorable for potency. Therefore, we directed our chemical optimization effort on this region via simple amide synthesis. We first investigated the growth inhibitory potency of these newly synthesized mifepristone derivatives in TNBC cell lines SUM149PT and HCC1937 using SRB assays. As the data shown in figure 1A, several new analogs, such as FZU-00,003 and FZU-00,004, displayed enhanced anticancer activities and suppressed cell viability more potently than MIF. Among all these derivatives, FZU-00,003 (Fig. 1B), had the most potent efficiency of inhibiting TNBC cell survival. Compared to MIF, the $\mathrm{IC}_{50}$ values of FZU-00,003 reduced from $17.2 \mu \mathrm{M}$ to $4.3 \mu \mathrm{M}$ and $11.3 \mu \mathrm{M}$ to $2.6 \mu \mathrm{M}$ for HCC1937 and SUM149PT, respectively.

In order to figure out whether FZU-00,003 could suppress survival of breast cancer cells other than HCC1937 and SUM149PT cell lines, we also treated 5 other breast cancer cell lines (including another TNBC cell line HCC1806, two luminal breast cancer cell lines MCF7 and T47D, and two HER2+ cell lines SKBR3 and BT474) with FZU-00,003. MIF was also tested for comparison. As shown in figure 1C, FZU-00,003 reduced cell viability at much lower concentrations than MIF in all cell lines tested. Overall, FZU-00,003 reduced breast cancer cell viability much more effectively than MIF did.

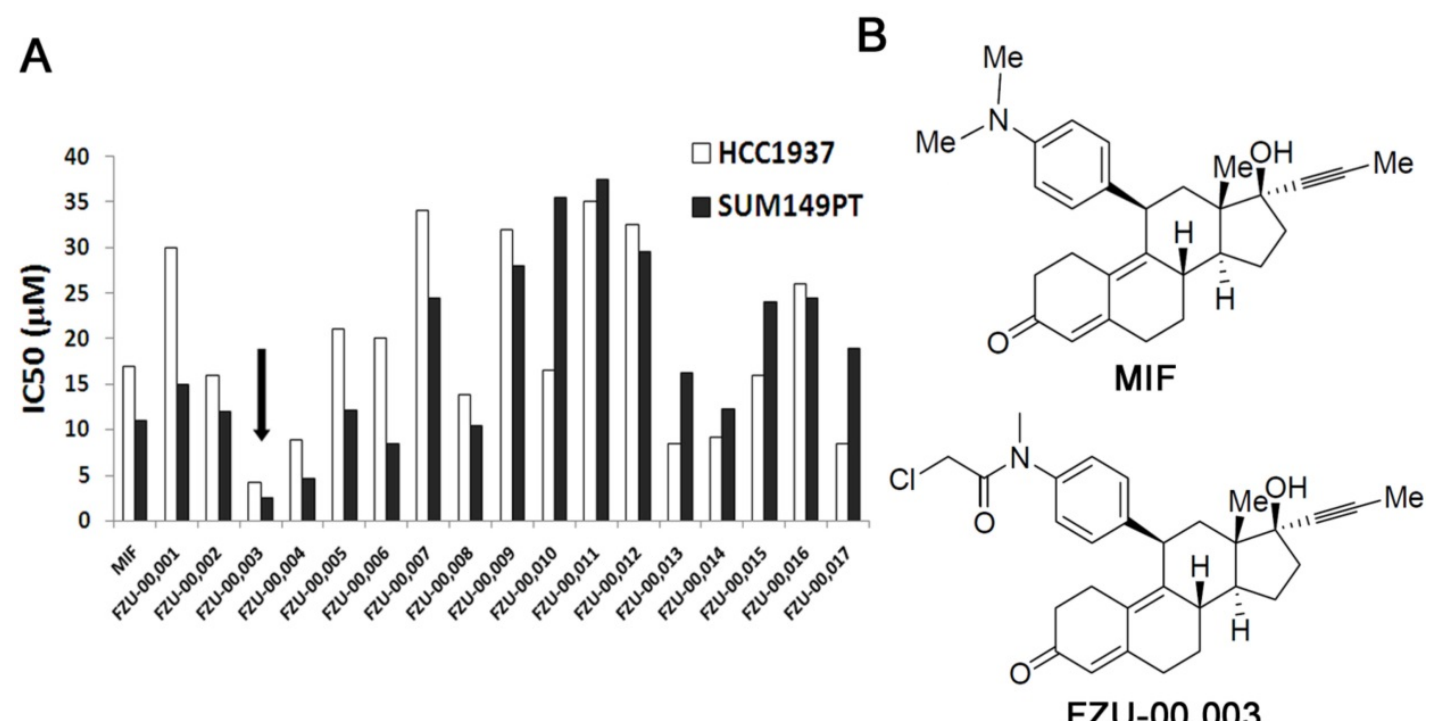

C
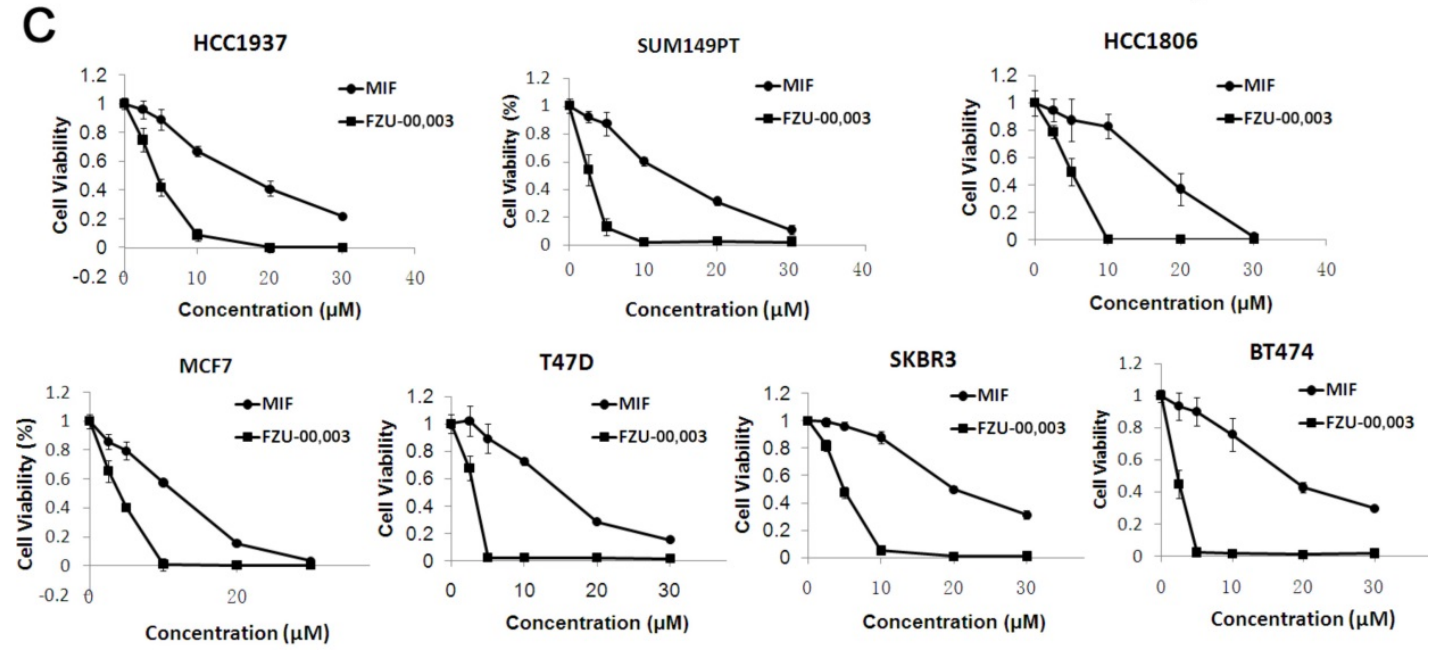

Figure 1. FZU-00,003 was identified as a more potent anti-cancer compound derived from mifepristone in breast cancer cells. A. Effects of new Mifepristone derivatives on cell survival of HCC1937 and SUM149PT TNBC cell lines. B. Chemical structures of MIF and FZU-00,003. C. The cytotoxicity of FZU-00,003 and MIF in 7 different breast cancer lines. The cells were treated with compounds at indicated dosages for 48 hours, and cell viability was measured using the SRB assay. 


\section{FZU-00,003 suppresses TNBC cell proliferation more potently than MIF}

It is well known that both cell growth inhibition and cell death result in reduction of cell viability. To determine the mechanism by which FZU-00,003 reduced cell viability better than MIF, we first tested whether FZU-00,003 inhibits cell growth through affecting cell proliferation. The EdU-incorporation assay was performed in HCC1937 and SUM149PT to detect the TNBC cell proliferation. As shown in figure $2 \mathrm{~A}$, the percentages of EdU-positive cells decreased in dosage-dependent manners in both TNBC cell lines when the cells were treated with FZU-00,003 and MIF. FZU-00,003 inhibited DNA synthesis in both cancer cell lines at much lower concentrations than MIF did. To confirm this result, we examined cell cycle progression. Consistently, FZU-00,003 inhibited cell cycle progression, especially G1/S progression, in a dosage-dependent manner (Fig. 2B). As expected, FZU-00,003 suppressed G1/S cell cycle progression at much low dosages compared to MIF.

Additionally, FZU-00,003 induced apoptosis was analyzed by the Annexin $\mathrm{V}$ staining. Flow cytometry analysis showed that FZU-00,003 induced apoptosis in both HCC1937 and SUM149PT cell lines in a dosage-dependent manner (Fig. 2C). FZU-00,003 significantly induced apoptosis at a concentration of 5 $\mu \mathrm{M}$ while MIF only induced moderate apoptosis at 10-20 $\mu \mathrm{M}$. These results implicated that the anticancer activity of FZU-00,003 is stronger than MIF.

\section{FZU-00,003 suppresses KLF5 expression more potently than MIF}

As we have demonstrated previously that MIF suppressed TNBC cell growth and survival partially through down-regulating the expression of KLF5 through inducing the expression of miR-153 [13], we then checked whether FZU-00,003 also suppresses KLF5 expression in TNBC cells. Indeed, FZU-00,003 suppressed KLF5 expression at much lower concentrations than MIF did in both HCC1937 and SUM149PT cell lines (Fig. 3A). Compared to MIF, FZU-00,003 induced more cell cycle dependent kinase inhibitor p21, whose transcription is well known to be suppressed by KLF5 [24]. Consistently, FZU-00,003 induced more apoptosis as evidenced by the induction of cleaved PARP than MIF did (Fig. 3A).

Since miR-153 is responsible for MIF to suppress KLF5 expression [13], we next investigated the effects of FZU-00,003 on miR-153 induction. As expected, FZU-00,003 induced more miR-153 at lower concentrations than MIF did (Fig. 3B).

\section{FZU-00,003 decreases cell viability partially via miR-153-KLF5 axis}

Since FZU-00,003 regulates miR-153-KLF5 axis and decreased cell viability in TNBC, we first tested whether FZU-00,003 decreased cell viability through down-regulating KLF5 expression. We overexpressed KLF5 in HCC1937 and treated the cells with FZU-00,003. Indeed, ectopic overexpression of KLF5 significantly reduced FZU-00,003-induced loss of cell viability and apoptosis indicated by PARP cleavage (Fig. 4A-B). Meanwhile, over-expression of KLF5 rescued the induction of p21 by FZU-00,003 (Fig. 4A). Meanwhile, we further validated whether FZU-00,003 inhibits the KLF5 expression and cell viability through inducing the miR-153. HCC1937 cells were transfected with miR-153 inhibitors followed by treating with FZU-00,003. Indeed, miR-153 inhibitors partially rescued MIF-induced KLF5 decrease, loss of cell viability and apoptosis indicated by PARP cleavage (Fig. 4C-D).

\section{FZU-00,003 suppresses TNBC cell growth in vivo}

FZU-00,003 suppressed TNBC cell growth and induced apoptosis in vitro, we then determined whether FZU-00,003 suppresses tumor growth in vivo. We established UM1 PDX xenografts and treated the mice with $1 \mathrm{mg} / \mathrm{d}$ FZU-00,003 or control for 3 weeks, MIF was also tested for comparison. As expected, FZU-00,003 significantly inhibited UM1 tumor growth better than MIF (Fig. 5A-C) in nude mice without affecting the body weight of the mice (Fig. 5D).

\section{Discussion}

As the most malignant subtype of breast cancers, TNBC has the poorest prognosis but does not have effective targeted therapies. We have previously demonstrated that MIF, a synthetic PR antagonist that has been safely used as an abortifacient and an emergency contraceptive for decades [21], has an anti-tumor activity in basal TNBC cells through inhibiting KLF5 expression at relatively high doses $(10-20 \mu \mathrm{M})$ [13]. A clinical trial revealed that the peak MIF plasma concentration is about $10 \mu \mathrm{M}$, which is close to the effective concentration we investigated in breast cancer cells in vitro, 1-2 hours and remains in the micromolar range for the next 24-48 hours after a single dose of $200 \mathrm{mg}$ MIF administration in health female volunteers [25]. Nevertheless, a Phase II study of MIF (200 mg daily) in 28 postmenopausal patients with PR-positive breast cancer suggested that only $10.7 \%$ of patients responded to MIF alone [26]. In order to improve the anticancer activity of MIF, we synthesized 17 mifepristone derivatives by modifying the methyl moiety in the sensitive metabolism region. 
A
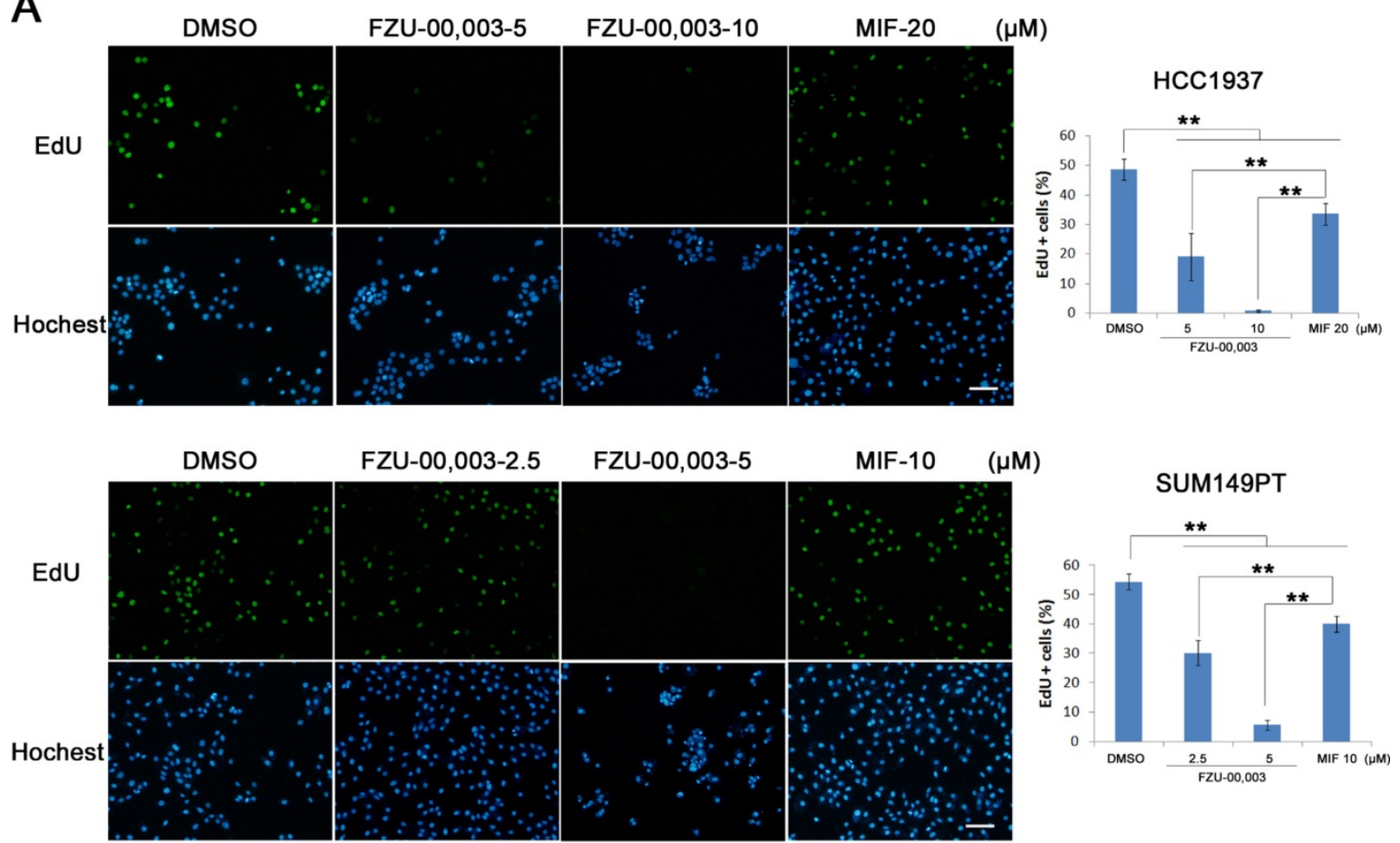

(scale bar: $100 \mu \mathrm{m}$ )

B

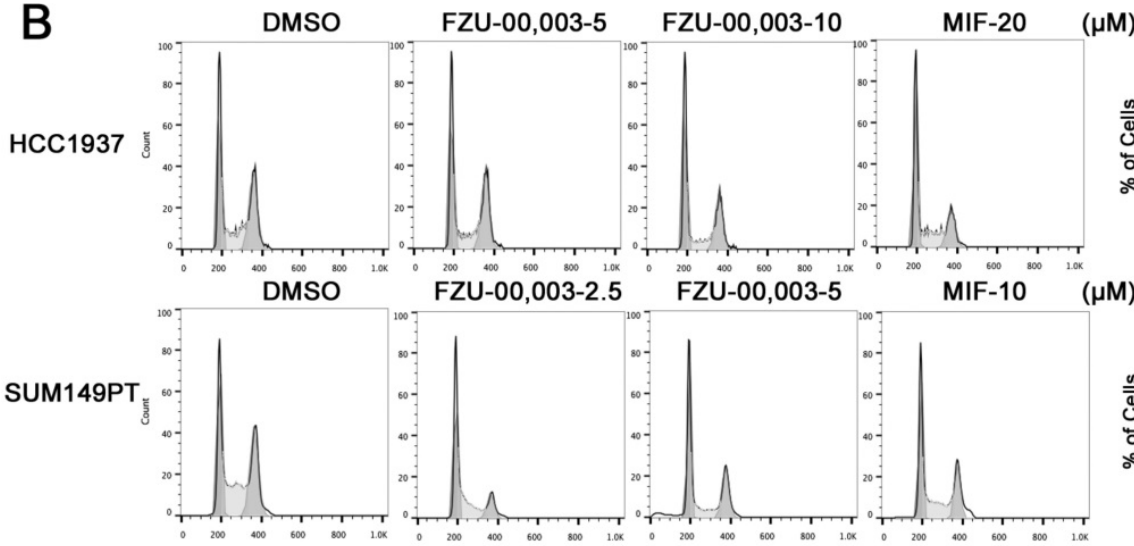

MCC1937

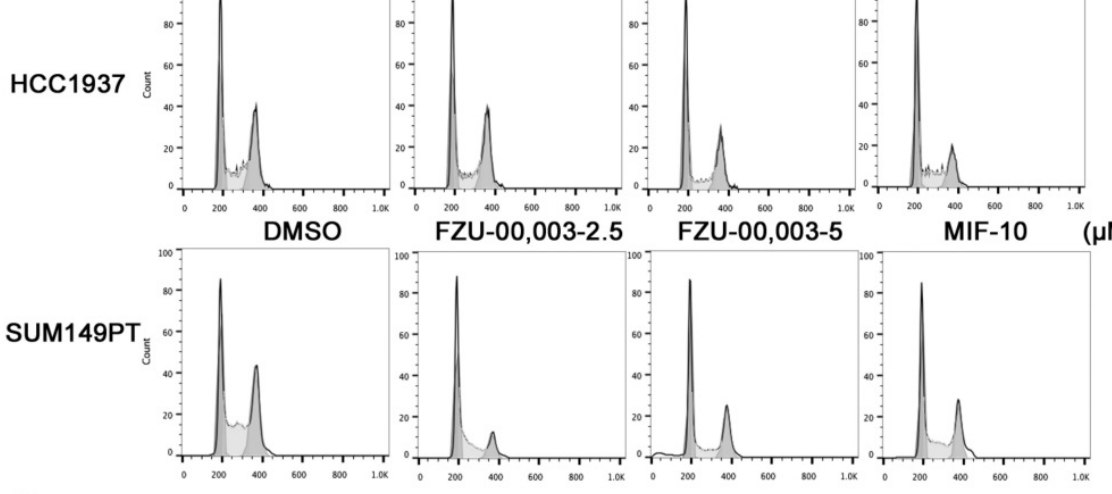

C
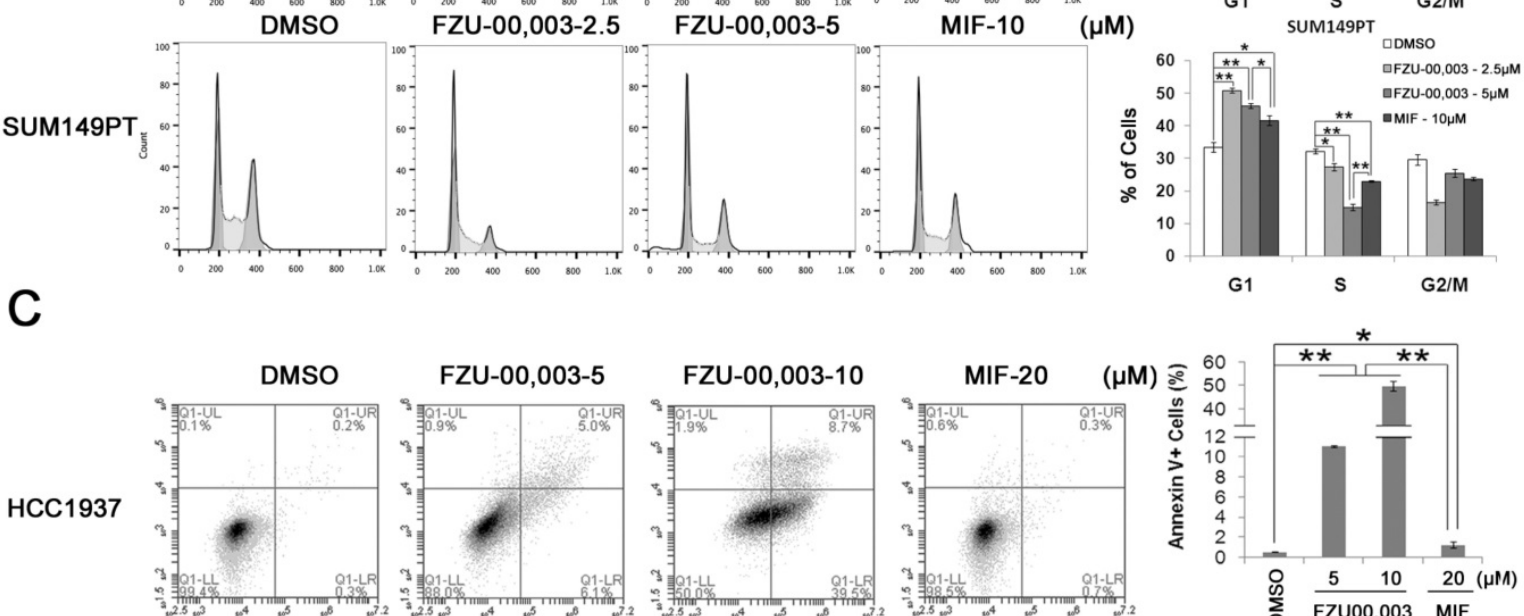

FZU-00,003-5

FZU-00,003-10
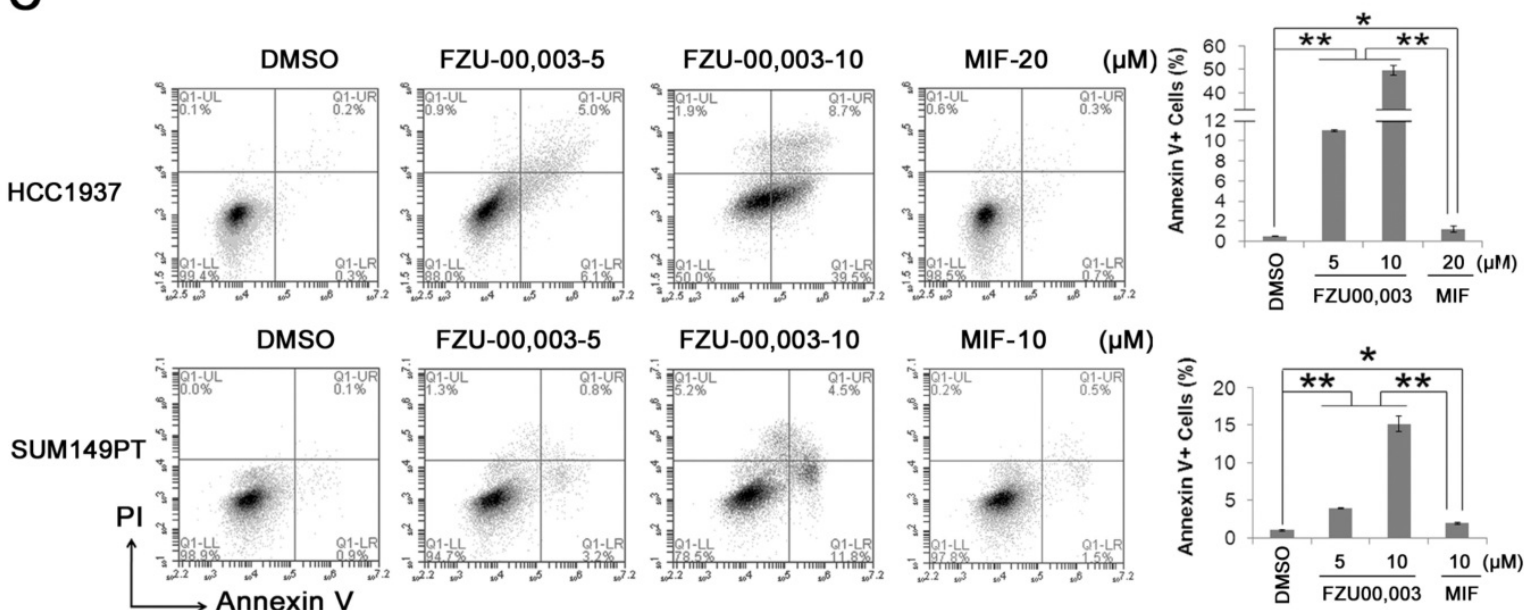

FZU-00,003-5
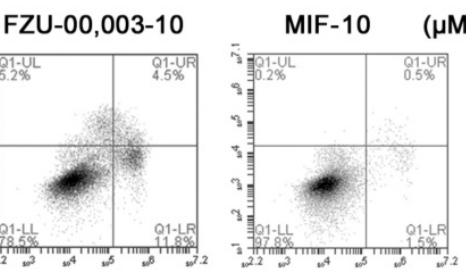

$(\mu \mathrm{M})$

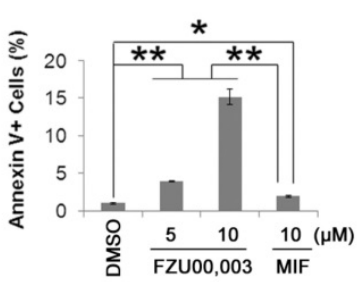

Figure 2. FZU-00,003 suppresses TNBC cell proliferation and promotes apoptosis. A. FZU-00,003 suppressed HCC1937 and SUM149PT cell proliferation more efficiently than MIF in a dosage-dependent manner. DNA synthesis of FZU-00,003 or MIF treated cells were examined using the Click-iTTM EdU Alexa Fluor ${ }^{\circledR} 488$ Imaging Kit. The quantitative results are shown on the right. B. FZU-00,003 inhibited HCC1937 and SUM149PT G1/S cell cycle progression more potently than MIF did. HCC1937 and SUM149PT cells were treated with FZU-00,003 or MIF for 24 hours. The cells were then collected and fixed for cell cycle analysis. C. FZU-00,003 induced more apoptosis in HCC1937 and SUM149PT than MIF did. HCC1937 and SUM149PT cells were treated with FZU-00,003 or MIF at indicated concentrations for 24 hours. The cells were then collected for Annexin $\mathrm{V}$ staining and flow cytometry analysis. *, $\mathrm{P}<0.05$, **, $\mathrm{P}<0.01$, t-test. 


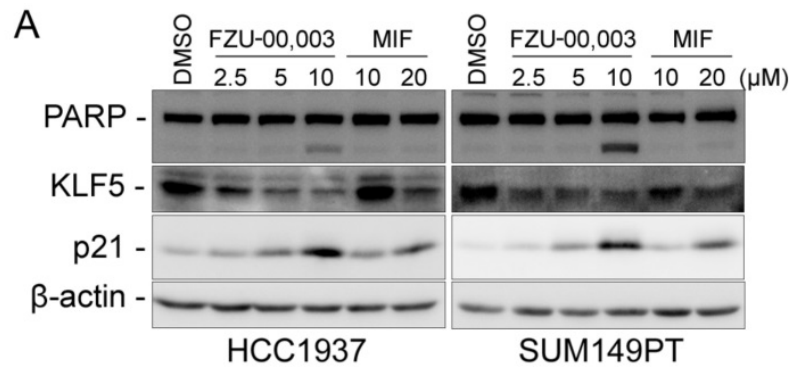

B

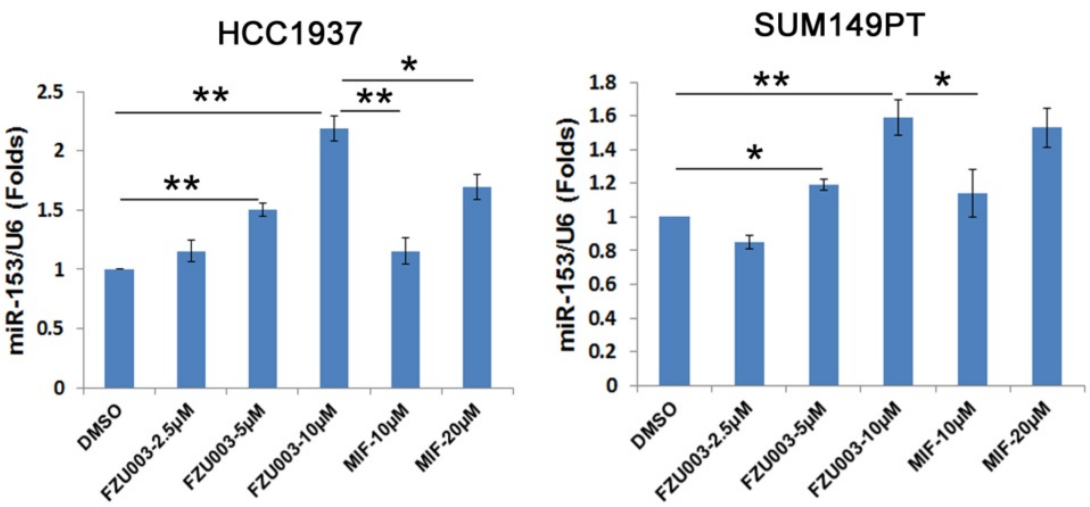

Figure 3. FZU-00,003 suppressed KLF5 expression via inducing the expression of miR-153 in TNBC cells. A. FZU-00,003 down-regulated KLF5 protein levels and induced the cleavage of PARP in a dosage-dependent manner in TNBC cells. Both HCC1937 and SUM149PT cells were treated with FZU-00,003 or MIF at indicated dosages for 24 hours. DMSO was added as the negative control. The cells were then collected for WB analysis. $\beta$-actin was used as the loading control. B. FZU-00,003 induced miR-153 expression more effectively than MIF did. FZU-00,003 or MIF treated cells were collected with Trizol for RNA extraction. Total RNA was then subjected to miRs reverse transcription and QPCR for detection of miR-153 expression. The expression of U6 was detected as the internal control.
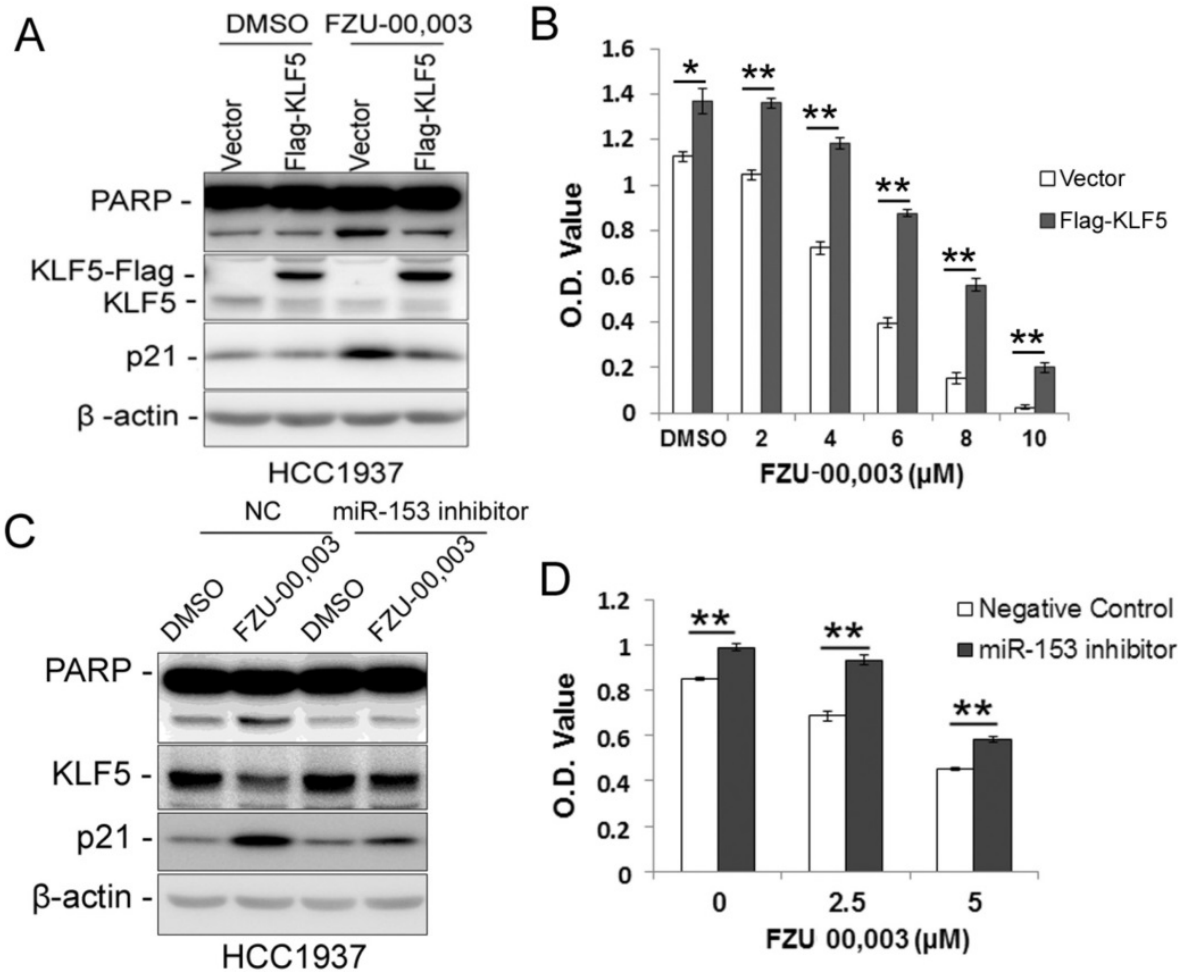

Figure 4. Ectopic over-expression of KLF5 partially rescues FZU-00,003 induced apoptosis and cell viability reduction in HCC1937. A. KLF5 over-expression decreases FZU-00,003-induced PARP cleavage in HCC1937. HCC1937 cells were infected with PCDH-Flag -KLF5 or vector control and treated with $5 \mu$ M FZU-00,003 for 24 hours. The apoptosis marker cl-PARP was detected by WB. B. Ectopic expression of KLF5 in HCC1937 partially rescued the FZU-00,003 induced cell viability reduction. $\mathrm{HCCl} 937$ cell were infected with PCDH-Flag-KLF5 or vector control and treated with FZU-00,003 at indicated concentrations for 48 hours before the cells were fixed for SRB assays. C. miR-153 inhibitor decreases FZU-00,003-induced KLF5 suppression and PARP cleavage in HCC1937. HCC1937 cells were transfected with miR-153 inhibitor or negative control and treated with $5 \mu$ M FZU-00,003 for 24 hours. D. miR-153 inhibitor partially rescued the FZU-00,003 induced cell viability reduction in HCC1937. $\mathrm{HCC} 1937$ cells were transfected with miR-153 inhibitor or negative control and treated with FZU-00,003 at indicated concentrations for 48 hours before the cells were fixed for SRB assays. *, $\mathrm{P}<0.05, * *, \mathrm{P}<0.01$, t-test. 
A
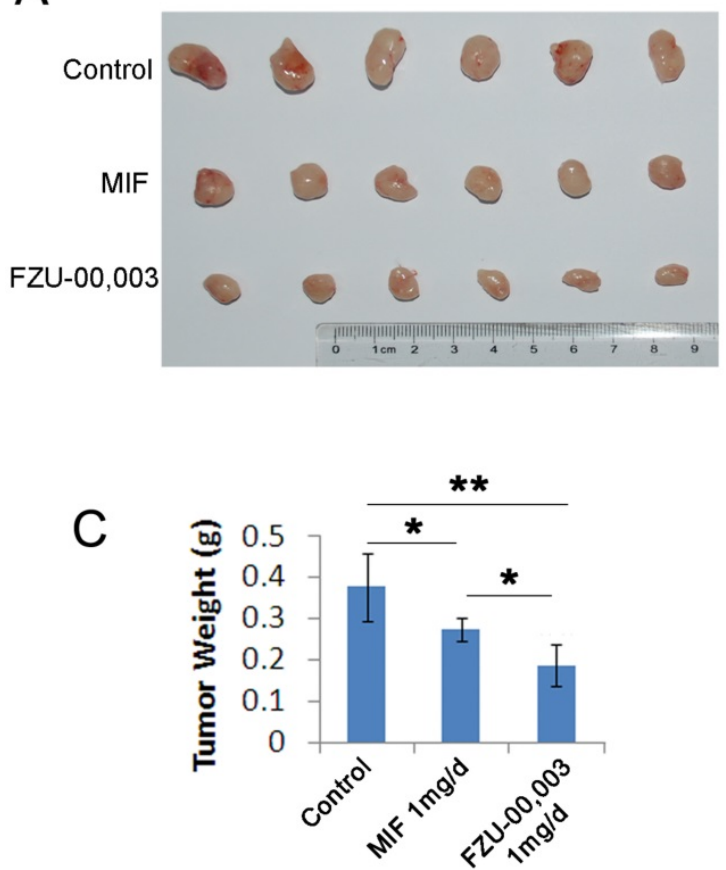

B

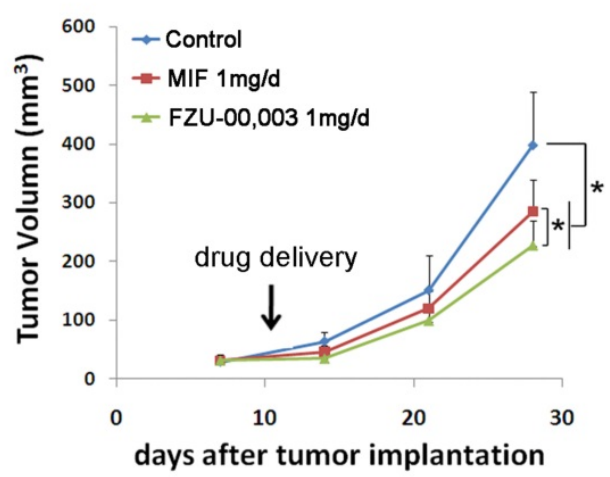

D

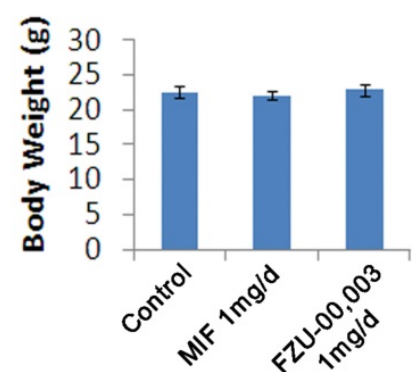

Figure 5. FZU-00,003 suppresses UMI xenograft tumor growth in vivo. A-B. FZU-00,003 suppressed UM1 tumor growth in Balb/c nude mice. UMI cells were injected into the fat pat of female Balb/c nude mice. When the average tumor size reached about $50 \mathrm{~mm}^{3}$ after inoculation, the mice were randomly and equally distributed into three groups ( $n=6 / g r o u p)$ : sesame oil control, $1 \mathrm{mg} / \mathrm{d}$ FZU-00,003 and $1 \mathrm{mg} / \mathrm{d}$ MIF. Tumor size was measured twice per week for 3 weeks. Tumors were collected 3 weeks after drug treatment. C. FZU-00,003 significantly decreased tumor weights compared to the MIF and control groups ( ${ }^{*}, \mathrm{p}<0.05$, $\left.{ }^{*}, \mathrm{p}<0.01, \mathrm{t}-\mathrm{test}\right)$. D. Both FZU-00,003 and MIF did not affect the body weight of the mice. The mice were weighed at the end of the experiment.

Remarkably, several newly synthesized mifepristone analogs have enhanced anticancer effects against two TNBC cell lines SUM149PT and HCC1937 compared to MIF. In this study, one of the novel MIF analogs FZU-00,003 was characterized in details. In comparison to MIF, FZU-00,003 (5-10 $\mu \mathrm{M})$ exhibited more notable growth inhibitory and apoptosis-promoting effects in SUM149PT and HCC1937 TNBC cell lines. Importantly, FZU-00,003 (1 $\mathrm{mg} / \mathrm{d}$ ) significantly suppressed TNBC PDX xenograft tumor (UM1) growth in vivo without affecting mouse body weight.

Our previous studies demonstrated that KLF5 is highly expressed in basal TNBC cell lines and depletion of KLF5 significantly inhibits TNBC xenograft growth in vivo [19]. Yagi et al delivered KLF5 siRNA into prostate cancer-bearing mice and significant suppressed PC-3 prostate tumor growth [27]. Bialkowska et al. identified two small molecules suppressing the KLF5 expression and significantly inhibited colorectal cancer cell proliferation [28]. More recently, our and other groups have reported that pharmacological inhibition of KLF5 by various inhibitors significantly suppressed cancer cell growth and/or survival. Curcumin suppresses bladder cancer cell growth through down-regulating KLF5 expression [29]. ML264, a small molecule inhibitor of KLF5, potently inhibits proliferation of colorectal cancer cells [30]. We recently reported metformin inhibits KLF5 expression and cancer stem cell in basal TNBC [14]. All these data suggest that KLF5 could serve as a therapeutic target for different cancers, including breast cancer, colon cancer, prostate cancer and bladder cancer.

FZU-00,003 more efficiently down-regulated KLF5 expression through inducing miR-153 in basal TNBC cell lines compared to MIF. Moreover, both ectopic over-expression of KLF5 and miR-153 inhibitor partially rescued FZU-00,003 caused reduction of cell viability in HCC1937 indicated that FZU-00,003, at least partially, suppressed TNBC cell survival through miR-153/KLF5 axis. Of course, we could not exclude the possibility that targets other than KLF5 are involved in the anti-TNBC functions of FZU-00,003, which still need to be investigated.

Besides TNBC cells, FZU-00,003 also showed strong survival inhibition effects in other subtypes of breast cancer (Fig 1C), indicating FZU-00,003 may also be effective in treating luminal and HER2 positive breast cancers through other mechanisms since KLF5 is lowly expressed in these subtypes of breast cancer cells [18]. Meanwhile, other cancers, including colon cancer, prostate cancer and bladder cancer, etc., with high KLF5 expression may also benefit from FZU-00,003 treatment. Although FZU-00,003 suppressed breast cancer cell survival at much lower dosages than MIF did, it was still used at micromole scale, implicating that further scaffold repurposing 
and structural optimization is still needed to obtain even more potent analogs in the future.

In conclusion, FZU-00,003 may serve as a better lead compound for the treatment of highly aggressive triple-negative breast cancers compared to MIF. Further anticancer mechanism investigation revealed that FZU-00,003 induces the expression of miR153 and inhibits KLF5 expression, like MIF but more efficiently. Preclinical studies will be needed to promote the clinical use of this compound in the future.

\section{Supplementary Material}

Supplementary tables.

http://www.ijbs.com/v16p0611s1.pdf

\section{Acknowledgement}

This study was supported in part by grants from the National Key R\&D Program of China (2018YFC 2000400), the National Natural Science Foundation of China $(81772847,81322038,81402781$, U1602221, U150 2222, 81401201, 81671458, 81830087, and 31771516), and Science and Technology Innovation Team of Yunnan Province (2019HC005).

\section{Competing Interests}

The authors have declared that no competing interest exists.

\section{References}

1. Lehmann BD, Bauer JA, Chen X, Sanders ME, Chakravarthy AB, Shyr Y, et al. Identification of human triple-negative breast cancer subtypes and preclinical models for selection of targeted therapies. J Clin Invest. 2011; 121: 2750-67.

2. Carey LA, Dees EC, Sawyer L, Gatti L, Moore DT, Collichio F, et al. The triple negative paradox: primary tumor chemosensitivity of breast cancer subtypes. Clinical cancer research : an official journal of the American Association for Cancer Research. 2007; 13: 2329-34.

3. Dent R, Trudeau M, Pritchard KI, Hanna WM, Kahn HK, Sawka CA, et al. Triple-negative breast cancer: clinical features and patterns of recurrence. Clinical cancer research : an official journal of the American Association for Cancer Research. 2007; 13: 4429-34.

4. Bianchini G, Balko JM, Mayer IA, Sanders ME, Gianni L. Triple-negative breast cancer: challenges and opportunities of a heterogeneous disease. Nat Rev Clin Oncol. 2016; 13: 674-90

5. Anders CK, Winer EP, Ford JM, Dent R, Silver DP, Sledge GW, et al. Poly(ADP-Ribose) polymerase inhibition: "targeted" therapy for triple-negative breast cancer. Clin Cancer Res. 2010; 16: 4702-10.

6. Imai K, Takaoka A. Comparing antibody and small-molecule therapies for cancer. Nat Rev Cancer. 2006; 6: 714-27.

7. Fouque A, Delalande O, Jean M, Castellano R, Josselin E, Malleter M, et al. A novel covalent mTOR Inhibitor, DHM25, shows in vivo antitumor activity against triple-negative breast cancer cells. J Med Chem. 2015; 58: 6559-73.

8. Shu S, Lin CY, He HH, Witwicki RM, Tabassum DP, Roberts JM, et al. Response and resistance to BET bromodomain inhibitors in triple-negative breast cancer. Nature. 2016; 529: 413-7.

9. Chen $\mathrm{CH}$, Yang N, Zhang Y, Ding J, Zhang W, Liu R, et al. Inhibition of super enhancer downregulates the expression of KLF5 in basal-like breast cancers. Int J Biol Sci. 2019; 15: 1733-42.

10. Fosu-Mensah N, Peris MS, Weeks HP, Cai J, Westwell AD. Advances in small-molecule drug discovery for triple-negative breast cancer. Future Med Chem. 2015; 7: 2019-39.

11. Liu R, Zheng HQ, Zhou Z, Dong JT, Chen C. KLF5 promotes breast cell survival partially through fibroblast growth factor-binding protein 1-pERK-mediated dual specificity MKP-1 protein phosphorylation and stabilization. J Biol Chem. 2009; 284: 16791-8.

12. Zheng HQ, Zhou Z, Huang J, Chaudhury L, Dong JT, Chen C. Kruppel-like factor 5 promotes breast cell proliferation partially through upregulating the transcription of fibroblast growth factor binding protein 1. Oncogene. 2009; 28 : 3702-13.

13. Liu R, Shi P, Nie Z, Liang H, Zhou Z, Chen W, et al. Mifepristone Suppresses Basal Triple-Negative Breast Cancer Stem Cells by Down-regulating KLF5 Expression. Theranostics. 2016; 6: 533-44.

14. Shi $\mathrm{P}$, Liu $\mathrm{W}$, et al. Metformin suppresses triple-negative breast cancer stem cells by targeting KLF5 for degradation. Cell Discov. 2017; 3: 17010.

15. Ben-Porath I, Thomson MW, Carey VJ, Ge R, Bell GW, Regev A, et al. An embryonic stem cell-like gene expression signature in poorly differentiated aggressive human tumors. Nature genetics. 2008; 40: 499-507.

16. Tong D, Czerwenka K, Heinze G, Ryffel M, Schuster E, Witt A, et al. Expression of KLF5 is a prognostic factor for disease-free survival and overall survival in patients with breast cancer. Clinical cancer research : an official journal of the American Association for Cancer Research. 2006; 12: 2442-8.

17. Takagi K, Miki Y, Onodera Y, Nakamura Y, Ishida T, Watanabe M, et al. Kruppel-like factor 5 in human breast carcinoma: a potent prognostic factor induced by androgens. Endocrine-related cancer. 2012; 19: 741-50.

18. Liu R, Zhou Z, Zhao D, Chen C. The induction of KLF5 transcription factor by progesterone contributes to progesterone-induced breast cancer cell proliferation and dedifferentiation. Mol Endocrinol. 2011; 25: 1137-44.

19. Zhao D, Zhi X, Zhou Z, Chen C. TAZ antagonizes the WWP1-mediated KLF5 degradation and promotes breast cell proliferation and tumorigenesis. Carcinogenesis. 2012; 33: 59-67.

20. Zhi X, Zhao D, Zhou Z, Liu R, Chen C. YAP promotes breast cell proliferation and survival partially through stabilizing the KLF5 transcription factor. The American journal of pathology. 2012.

21. Spitz IM. Mifepristone: where do we come from and where are we going? Clinical development over a quarter of a century. Contraception. 2010; 82: 442-52.

22. Li W, Zhang H, Nie M, Tian Y, Chen X, Chen C, et al. Ursolic acid derivative FZU-03,010 inhibits STAT3 and induces cell cycle arrest and apoptosis in renal and breast cancer cells. Acta Biochim Biophys Sin 2017; 49: 367-73.

23. Chen C, Sun X, Ran Q, Wilkinson KD, Murphy TJ, Simons JW, et al. Ubiquitin-proteasome degradation of KLF5 transcription factor in cancer and untransformed epithelial cells. Oncogene. 2005; 24: 3319-27.

24. Wang C, Nie Z, Zhou Z, Zhang H, Liu R, Wu J, et al. The interplay between TEAD4 and KLF5 promotes breast cancer partially through inhibiting the transcription of p27Kip1. Oncotarget. 2015; 6: 17685-97.

25. Sitruk-Ware R, Spitz IM. Pharmacological properties of mifepristone: toxicology and safety in animal and human studies. Contraception. 2003; 68: 409-20.

26. Perrault D, Eisenhauer EA, Pritchard KI, Panasci L, Norris B, Vandenberg T, et al. Phase II study of the progesterone antagonist mifepristone in patients with untreated metastatic breast carcinoma: a National Cancer Institute of Canada Clinical Trials Group study. J Clin Oncol. 1996; 14: 2709-12.

27. Yagi N, Manabe I, Tottori T, Ishihara A, Ogata F, Kim JH, et al. A nanoparticle system specifically designed to deliver short interfering RNA inhibits tumor growth in vivo. Cancer research. 2009; 69: 6531-8.

28. Bialkowska AB, Crisp M, Bannister T, He Y, Chowdhury S, Schurer S, et al. Identification of small-molecule inhibitors of the colorectal cancer oncogene Kruppel-like factor 5 expression by ultrahigh-throughput screening. Molecular cancer therapeutics. 2011; 10: 2043-51.

29. Gao Y, Shi Q, Xu S, Du C, Liang L, Wu K, et al. Curcumin promotes KLF5 proteasome degradation through downregulating YAP/TAZ in bladder cancer cells. International journal of molecular sciences. 2014; 15: 15173-87.

30. Ruiz de Sabando A, Wang C, He Y, Garcia-Barros M, Kim J, Shroyer KR, et al. ML264, A Novel Small-Molecule Compound That Potently Inhibits Growth of Colorectal Cancer. Molecular cancer therapeutics. 2016; 15: 72-83. 